\title{
Amoebic liver abscesses with an unusual source: a case report
}

\author{
Juanita N Chui', BSc (Adv), MD, Albert KK Chui ${ }^{2}$, FRACS, MD \\ ${ }^{1}$ School of Medicine, University of Sydney, Sydney, Australia \\ ${ }_{2}^{2}$ Private Practice, 12/F, Emperor Commercial Centre, Central, Hong Kong \\ *Corresponding author: akkchui@netvigator.com
}

Hong Kong Med J 2021;27:450-1

https://doi.org/10.12809/hkmj209157

\section{Case report}

In July 2020, a 50-year-old man presented to a public hospital in Hong Kong with a 3-week history of abdominal pain localising to the right upper quadrant, associated with low-grade fever, chills,

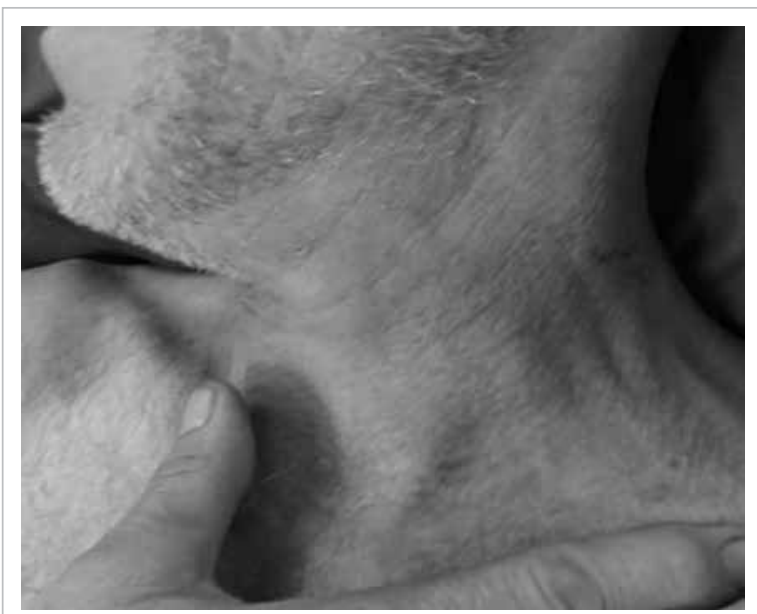

FIG I. A 50-year-old man presented with a 3-week history of abdominal pain localising to the right upper quadrant, associated with low grade fever, chills, and fatigue. The patient was bitten by a horse I month before onset of symptoms. Clinical photograph of the bite wounds on the neck after blood cleansing and fatigue. He denied any diarrhoea or vomiting. His medical history and family history were unremarkable. He was a non-smoker and consumed alcohol infrequently. He had recently returned from Southern California, United States, where he was bitten by a horse 1 month before the onset of his symptoms. Despite sustaining deep wounds to his left neck (Fig 1), he did not seek medical attention at the time.

On admission, a contrast-enhanced computed tomography (CT) scan of the abdomen revealed three liver abscesses. He was treated initially with intravenous antibiotic (amoxicillin/clavulanic acid for 5 days before switching to piperacillin/ tazobactam) but his clinical condition showed no improvement. A new CT scan 5 days later showed enlargement of the abscesses. Percutaneous drainage of the abscesses was performed under ultrasound guidance and the patient was transferred to our private hospital for further management.

On arrival, the patient was haemodynamically stable but clinically dehydrated, jaundiced, and delirious. The abdomen was soft and non-tender, with three abdominal drainage tubes in situ. A new CT scan confirmed that the liver abscesses and the drainage tubes were blocked, and they had to be replaced (Fig 2). Drainage fluid afterwards was noted to have the appearance of anchovy sauce and amoebic infection was suspected.

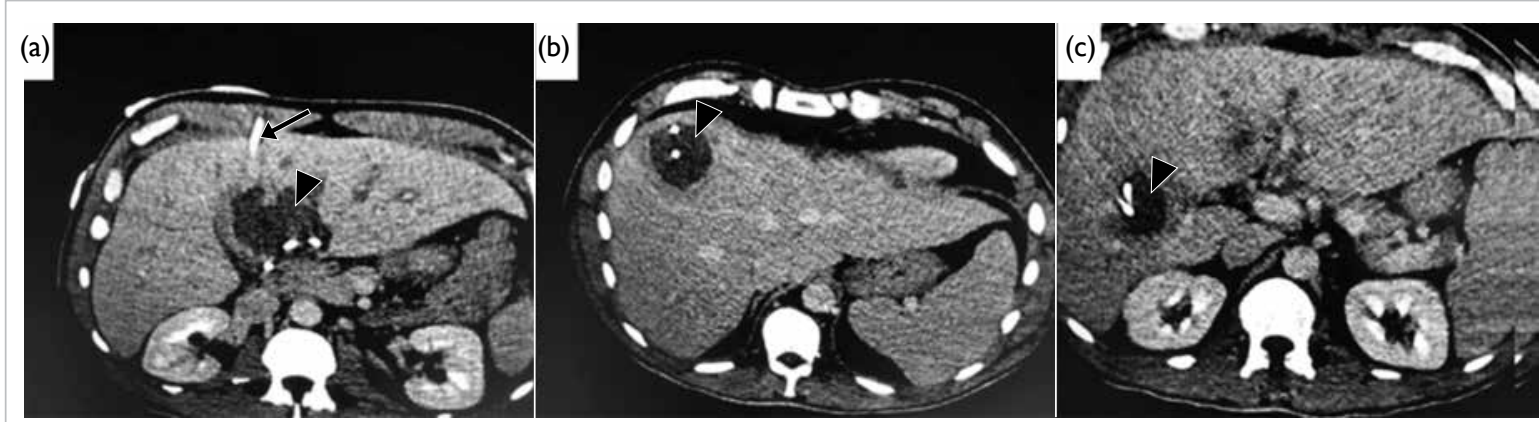

FIG 2. Contrast-enhanced computed tomography scans of the patient's liver after drainage, showing (a) the segment IV abscess $(5.9 \times 4.9 \times 4.2 \mathrm{~cm})$ with indwelling drainage catheter, (b) the segment $\mathrm{VIII}$ abscess $(4 \times 4.3 \times 4.2 \mathrm{~cm})$, and $(\mathrm{c})$ the segment $\mathrm{V}$ abscess $(3.1 \times 3.7 \times 2.9 \mathrm{~cm})$ 
Blood tests on arrival showed leucocytosis (white cell count $31 \times 10^{9} / \mathrm{L}$ ) with markedly elevated inflammatory markers (C-reactive protein $357 \mathrm{mg} / \mathrm{L}$ ), and deranged liver function (alkaline phosphatase $231 \mathrm{U} / \mathrm{L}$, alanine aminotransferase $64 \mathrm{U} / \mathrm{L}$, bilirubin $56 \mu \mathrm{mol} / \mathrm{L}$ and albumin $26.5 \mathrm{~g} / \mathrm{L}$ ). Blood cultures were negative. No abnormalities were found on colonoscopy and stool ova, cysts and parasite microscopy were negative. Fluid drained from the abscesses was negative on microscopy and culture, but positive for Entamoeba histolytica DNA on polymerase chain reaction. Initial amoebic serology was negative for E histolytica immunoglobulin G antibodies. Intravenous metronidazole was commenced empirically in addition to piperacillin/ tazobactam. The patient subsequently made a steady recovery. New amoebic serology demonstrated a significant titre of $E$ histolytica immunoglobulin G antibodies 9 days later, indicating recent amoebic infection. Repeated blood tests showed continued normalisation of initial results and follow-up CT scans showed progressive resolution of abscesses. The patient was discharged from the hospital after 2 weeks.

\section{Discussion}

Amoebiasis is a leading parasitic infection in terms of morbidity and mortality worldwide. It is most prevalent in developing countries and in tropical regions. It is caused by the protozoa, E histolytica, transmitted via the faecal-oral route by ingestion of contaminated food or water containing amoebic trophozoites or cysts. Acute infection typically presents as amoebic colitis. The most common symptoms associated with amoebiasis are abdominal pain (98\% of cases), fever (74\%), and dysentery (30\%). ${ }^{1,2}$ Although extraintestinal complications of invasive infection are rare $(<1 \%$ of cases), liver abscesses are the most common secondary manifestation, occurring when trophozoites invade the colonic mucosa and penetrate mesenteric venules to enter the portal circulation. ${ }^{3}$

This case was an unusual presentation of amoebic liver abscess, confirmed by polymerase chain reaction testing of aspirates and serology results that suggested an acute infection. However, the patient presented with no gastrointestinal symptoms typical of amoebic colitis and stool investigations and colonoscopy were normal. The typical period of incubation for amoebiasis in those with liver abscesses has been reported to be 8 to 20 weeks. ${ }^{4}$ In this case, the patient presented with symptoms of invasive disease 4 weeks following his horse bite. The patient denied recent travel to an endemic area or any sick contacts. Southern California is known to have many migrants from South America where amoeba is prevalent. There was little evidence from the patient's clinical history or investigations to support a faecal-oral route of transmission. As such, the possibility of liver abscess from a cutaneous source was considered.

The patient was initially treated for pyogenic liver abscess. Drainage of the abscesses and the addition of metronidazole dramatically improved his condition. It is conceivable that the horse bite harboured amoebic trophozoites or otherwise facilitated their invasion from contaminated environmental water, soil, or vegetation. Just as invasive trophozoites are known to reach the liver by hematogenous dissemination to form abscesses, in our patient they may have entered the circulation via the bite wound to invade the liver. The short incubation period was also consistent with direct inoculation. Although cutaneous bacterial infection leading to liver pyogenic abscesses is well reported in the literature, the development of amoebic liver abscesses from a similar source has not previously been described. This may be the first described case of amoebic liver abscesses of cutaneous origin and warrants further study.

\section{Author contributions}

Concept or design: AKK Chui. Acquisition of data: AKK Chui. Analysis or interpretation of data: AKK Chui. Drafting of the manuscript: Both authors.

Critical revision of the manuscript for important intellectual content: Both authors.

All authors had full access to the data, contributed to the study, approved the final version for publication, and take responsibility for its accuracy and integrity.

\section{Conflicts of interest}

As an editor of the Journal, AKK Chui was not involved in the peer review process for this article. The other author has disclosed no conflicts of interest.

\section{Funding/support}

This study received no specific grant from any funding agency in the public, commercial, or not-for-profit sectors.

\section{Ethics approval}

The patient was treated in accordance with the tenets of the Declaration of Helsinki. Patient consent was obtained.

\section{References}

1. Akgün Y, Taçyulıdız ÏH, Çelik Y. Amoebic liver abscess: changing trends over 20 years. World J Surg 1999;23:1026.

2. Donovon AJ, Yellin AE, Ralls PW. Hepatic abscess. World J Surg 1991;15:162-9.

3. Swaminathan V, O'Rourke J, Gupta R, Kiire CF. An unusual presentation of an amoebic liver abscess: the story of an unwanted souvenir. BMJ Case Rep 2013;2013:bcr2012006964.

4. Li E, Stanley SL Jr. Protozoa. Amebiasis. Gastroenterol Clin North Am 1996;25:471-92. 